\title{
Long-lasting modifications of saccadic eye movements following adaptation induced in the double-step target paradigm
}

\author{
Nadia Alahyane and Denis Pélisson ${ }^{1}$ \\ Espace et Action, INSERM/Université Claude Bernard-Lyon, IFR19 Institut Fédératif des Neurosciences de Lyon, \\ 69676 Bron cedex, France
}

\begin{abstract}
The adaptation of saccadic eye movements to environmental changes occurring throughout life is a good model of motor learning and motor memory. Numerous studies have analyzed the behavioral properties and neural substrate of oculomotor learning in short-term saccadic adaptation protocols, but to our knowledge, none have tested the persistence of the oculomotor memory. In the present study, the double-step target protocol was used in five human subjects to adaptively decrease the amplitude of reactive saccades triggered by a horizontally-stepping visual target. We tested the amplitude of visually guided saccades just before and at different times (up to 19 days) after the adaptation session. The results revealed that immediately after the adaptation session, saccade amplitude was significantly reduced by $22 \%$ on average. Although progressively recovering over days, this change in saccade gain was still statistically significant on days 1 and 5, with an average retention rate of $36 \%$ and $19 \%$, respectively. On day 11 , saccade amplitude no longer differed from the pre-adaptation value. Adaptation was more effective and more resistant to recovery for leftward saccades than for rightward ones. Lastly, modifications of saccade gain related to adaptation were accompanied by a decrease of both saccade duration and peak velocity. A control experiment indicated that all these findings were specifically related to the adaptation protocol, and further revealed that no change in the main sequence relationships could be specifically related to adaptation. We conclude that in humans, the modifications of saccade amplitude that quickly develop during a double-step target adaptation protocol can remain in memory for a much longer period of time, reflecting enduring plastic changes in the brain.
\end{abstract}

Motor learning allows us to improve our motor performance to cope with new behavioral goals (skill learning) or with new sensori-motor conditions imposed by our changing environment (adaptation). Recently, motor adaptation has been studied in the case of reaching movements of the limb performed in a forcefield (Shadmehr and Brashers-Krug 1997; Shadmehr and Holcomb 1997) or submitted to visual perturbations (Fernandez-Ruiz and Diaz 1999; Tong and Flanagan 2003; Caithness et al. 2004) or in the case of the vestibulo-ocular reflex (VOR) in response to altered visuo-vestibular interactions (Kuki et al. 2004). Adaptation of saccadic eye movements represents another form of motor learning involved in the compensation for the physiological (growing, aging, fatigue) or pathological changes affecting the oculomotor system throughout life. Thus, when a saccadic eye movement becomes inaccurate because it is too small (hypometric) or too large (hypermetric) relative to the target position, saccadic adaptation mechanisms are elicited to gradually adjust the saccade gain (ratio of saccade amplitude to target eccentricity) such that the eyes again reach precisely the target. This phenomenon has been clearly revealed in patients and in monkeys with weakened extraocular muscles (Kommerell et al. 1976; Abel et al. 1978; Optican and Robinson 1980). These three studies report that the saccade hypometria of the weakened eye was progressively compensated for over successive days by an adaptive increase of the saccade gain toward normal values. Saccadic adaptation can also be induced in the laboratory in a noninvasive way by using the double-step target protocol described by

\footnotetext{
'Corresponding author.

E-mail pelisson@lyon.inserm.fr; fax 33-472-91-34-01.

Article and publication are at http://www.learnmem.org/cgi/doi/10.1101/ Im.96405.
}

McLaughlin (1967). This protocol consists first in eliciting a saccade in response to the appearance of a visual target and then, during the primary saccade, in displacing the target to create a post-saccadic visual error, without conscious perception by the subject. The repetition of such trials with either a backward or a forward intrasaccadic target step leads to a progressive reduction of the post-saccadic error through a decrease or an increase of the primary saccade amplitude, respectively. The adaptation elicited in the double-step target paradigm is faster than that observed under conditions of extraocular muscles weakness, since it takes only a few hundred trials in human and 1000-3000 trials in monkey. But in fact, as demonstrated by Scudder et al. (1998) in the monkey, a similar time course of adaptation is observed when the complexity of the set of error signals is equated in the two paradigms. This type of results has led to suggest that saccade adaptive modifications produced in the two different paradigms share similar properties and neural substrate. Another advantage of saccadic adaptation studied in the double-step target paradigm is that neither the visual target perturbation nor the elicited corrective response (corrective saccade) leads to conscious experience from the subject. Thus, the behavioral changes induced in this adaptation protocol largely reflect the true adaptive neural changes independently of any conscious strategic response. This contrasts with the other types of motor adaptation reviewed above in which subjects can use visual or proprioceptive feedback to consciously detect the experimentally induced perturbation and may thus try to deliberately adjust their motor responses.

Many studies have used the double-step target paradigm to investigate the mechanisms underlying saccadic adaptation and have discovered a number of functional and neurophysiological properties (for review, see Hopp and Fuchs 2004). It has been 
clearly shown in both man and monkey that adaptation of a single saccade transfers to all saccades of the same direction and amplitude regardless of their start and end positions in the orbit (vector specificity) (Miller et al. 1981; Deubel et al. 1986; Deubel 1987; Frens and Van Opstal 1994; Albano 1996; Straube et al. 1997; Noto et al. 1999; Watanabe et al. 2000). Thus, adaptation of leftward saccadic vectors does not transfer to rightward vectors, and vice versa. Saccadic adaptation also depends on the time when the visual error signal becomes available after the primary saccade (temporal specificity). Indeed, in both monkey and human (Bahcall and Kowler 2000; Shafer et al. 2000; Fujita et al. 2002), varying the time of occurrence of the second target step relative to the primary saccade revealed that saccadic adaptation is maximal for a narrow post-saccadic time window and declines sharply as the delay increases until reaching a nonsignificant level for delays $>1 \mathrm{sec}$. Furthermore, saccadic adaptation in humans depends on the experimental tasks to elicit saccades (saccade type specificity), as there is no or only a limited transfer of adaptation between reactive saccades triggered by the sudden appearance of a novel target and voluntary saccades directed to a predetermined target (e.g., scanning saccades). This suggests that the mechanisms and neural substrate of adaptation differ for these two saccade types (Erkelens and Hulleman, 1993; Deubel 1995a,b; Fujita et al. 2002). Finally, although the neural substrate of saccadic adaptation remains to be completely elucidated, there is a large body of evidence for an involvement of the cerebellar vermis and fastigial nucleus in the adaptation of reactive saccades, both in monkey (Optican and Robinson 1980; Barash et al. 1999) and in human (Desmurget et al. 1998; Straube et al. 2001).

Saccadic adaptation is supposed to elicit enduring changes in the neural circuits involved in saccade generation. However, contrary to the long-term storage of adaptive behavioral modifications that has been shown for the other sensorimotor systems cited above, the retention of saccadic adaptation has never been specifically investigated. Indeed, most studies of saccadic adaptation have focused on the rate and total amount of gain changes observed during the exposure phase, disregarding how long the effects of this initial learning are kept in memory. The only cues available in the literature concerning the retention of saccadic adaptation are indirect and based on measures of saccade gain recovery during de-adaptation. It has been shown in human (Deubel et al. 1986) and in monkey (Straube et al. 1997) that the rate of de-adaptation in the presence of a visual feedback provided by stationary visual targets is roughly as fast as that of adaptation, suggesting that the retention of adaptation is very restricted. However as envisioned by Deubel (1995a,b), because of the specificity of adaptive mechanisms relative to the different saccade types in humans, the natural recovery from adaptation of reactive saccades may be much longer than that evaluated in de-adaptation experiments because subjects mostly perform voluntary saccades in their daily activities.

Our study was thus aimed at measuring the duration of the after-effects following the adaptation of reactive saccades, in other terms the retention of adaptation over successive days. We induced a backward adaptation in five human subjects by using the double-step target protocol. We measured the saccade gain at different times immediately and up to $19 \mathrm{~d}$ after training. The subjects underwent normal visual experience between the different measures. Furthermore, we compared the efficiency and retention of adaptation of rightward versus leftward saccades and investigated the effect of adaptation on the saccade dynamics (main sequence) and latency. Preliminary results have been reported in a short article (Alahyane and Pélisson 2005).

\section{Results}

Subjects underwent a double-step target protocol to adaptively decrease the gain of their saccades. This learning phase (adaptation session) was composed of 440 double-step trials divided into 10 successive blocks (two A blocks, followed by two B blocks, followed by six C blocks) that differed in terms of amplitude of the first and second intrasaccadic target steps (for details, see Materials and Methods). A test session consisting of 40 single target steps was performed before the adaptation session (pretest), to measure the initial saccade performances, and at different times after the adaptation session (post-tests), to measure the effect of saccadic adaptation. On day 0 , each subject performed a pre-test (D0pre), a first post-test (DOa) following half of the adaptation session, and a second post-test (D0b) after the second half of the adaptation session. A post-test was then run on days $1,5,11$, and 19 (D1, D5, D11, D19, respectively). Between the different post-tests following the D0 session, subjects were free and pursued their daily activities normally. To know if the observed changes of saccade parameters were exclusively due to adaptation or if fatigue possibly accumulating after the large number of trials could also play a role, we performed a control experiment. This control experiment contained the same number of trials and the same successive sessions as did the main experiment. However during the adaptation session, no backward target step was introduced during the saccade. This "pseudo-adaptation" session was preceded by a pre-test and followed by post-tests at D0, D1, and D5. We begin by describing the effect of the double-step target paradigm on the gain of saccades (first, irrespective of saccade direction; second, in relation to saccade direction), followed by the presentation of saccade dynamics and latency.

\section{Effect of the double-step target protocol on the saccade gain}

\section{The two saccade directions combined}

\section{Main experiment}

Figure $1 \mathrm{~A}$, shows the relationship between the gain of saccades and the number of trials in the adaptation session for subject $B$ (day 0). The saccade gain progressively decreased over trials as a consequence of the backward intrasaccadic step introduced during the saccade. The gain decrease following the adaptive learning was retained since the saccade gain was smaller in the posttest performed after completion of the entire adaptation session (D0b), than the gain in the pre-test. The other four subjects exhibited the same pattern of results (data not shown). We plotted in Figure 2 the saccade gain as a function of the test sessions in each subject (A-E). We compared the saccade gain between the different test sessions by individual analyses of variance. We found a significant main effect of the test session factor in all subjects $(P<0.001)$. The individual post-hoc LSD Fisher tests indicated that all subjects exhibited a decrease in gain at DOa and D0b with respect to the pre-test gain $(P<0.001)$. When the data from the five subjects were pooled together (mean gain shown as a black trace in Fig. 2), the one-way repeated-measures ANOVA with "test session" as factor showed a significant main effect $\left(F_{(6,24)}=35.1 ; P<0.001\right)$. From a nearly unity value in the pre-test $(0.98 \pm 0.03)$, the gain had significantly decreased after the first half of the adaptation session (D0a; post-hoc Fisher LSD tests; $P<0.001$ ) and had decreased further after the second half of the adaptation session (DOb), reaching a significantly lower level than at D0pre $(P<0.001)$ and than at D0a $(P<0.01)$. The percentage of gain change calculated from these data revealed a mean gain reduction of $-16.4 \% \pm 4.6 \%$ at the

\section{Learning \& Memory}

www.learnmem.org 
A- Main experiment (subject B)

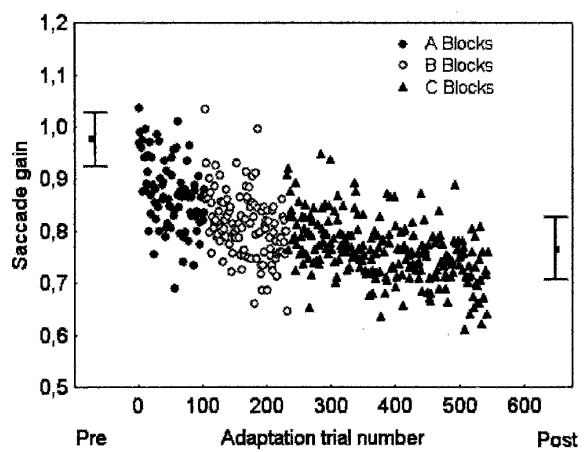

B- Control experiment (subject B)

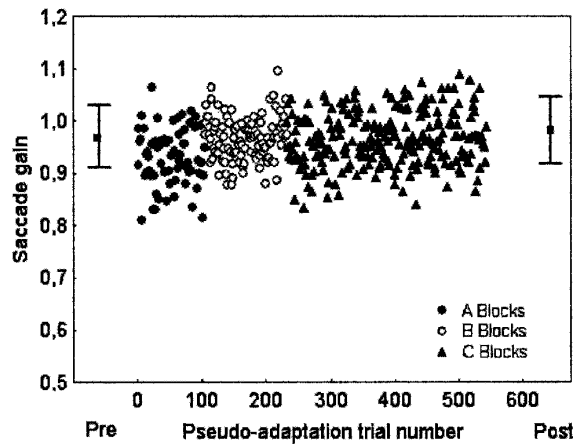

Figure 1. Time course of the saccade gain during the adaptation session of the main experiment $(A)$ and during the pseudo-adaptation session of the control experiment $(B)$ in subject $B$. The adaptation session consisted of three types of blocks of double-step trials differing in the amplitude of the intrasaccadic backward target step (step-2) relative to the first target step (step-1). They were presented in the following order: two A blocks (filled circles), then two B blocks (open circles), and finally six $\mathrm{C}$ blocks (filled triangles) (step- 2 amplitude $=25 \%, 33 \%$, and $40 \%$ respectively). The pseudo-adaptation contained the same successive blocks as in the adaptation session but without intrasaccadic target step (see Materials and Methods). The mean gain in pre-test session (Pre) and in the post-test session performed after completion of the entire adaptation or pseudo-adaptation session (Post) are also represented. Error bars, SD.

intermediate test session (D0a) and of $-22.1 \% \pm 4.7 \%$ at the end of adaptation (DOb). Thus, these results indicate that the adaptation protocol was successful in producing a significant backward adaptation of the horizontal saccades with a larger after-effect reached after all adaptation trials were completed.

We then studied how long the adaptive changes persisted over successive days after the adaptation session. The individual data illustrated in Figure 2 revealed that the persistence of the saccade hypometria was observed for all subjects, excluding subject D at D1 and excluding subjects A and D at D5. Concerning now the mean gain averaged across the five subjects, Figure 2 (upper left panel, black trace) shows that the mean gain began to increase as early as the following day, relative to D0b, but was still significantly smaller than the D0pre gain (post-hoc Fisher LSD tests; $P<0.001)$. At D5, saccades were still hypometric relative to those recorded before adaptation $(P<0.05)$. Thereafter, both at D11 and at D19, the gain returned to a level close to that obtained in pre-test $(P=0.11$ and $P=0.47$, respectively). In addition, the post-hoc analysis indicated that the gains at D5, D11, and D19 did not statistically differ from each other $(P>0.15)$. To quantify the level of maintenance of the adaptation induced at D0, we computed the amount of retention (see Materials and Methods). Figure 3 indicates that all subjects exhibited a relatively high amount of retention at D1, except subject D whose saccade gain was already back to a normal value, as already observed in Figure 2 . On average, the amount of retention of adaptation reached $36 \% \pm 17 \%$ at D1. It decreased to $19.7 \% \pm 13.3 \%$ at $* * * P<0.001$. test session $(P<0.001$; see previous paragraph) but no significant effect of the amplitude factor $(P=0.148)$. Moreover, there was no interaction between the two factors $\left(F_{(6,24)}=0.272 ; P=0.94\right)$. In fact, the contrast analysis indicated that the difference between the $10^{\circ}$ and $20^{\circ}$ amplitudes was only significant at D0a $(P<0.05)$,

Time course of saccade gain
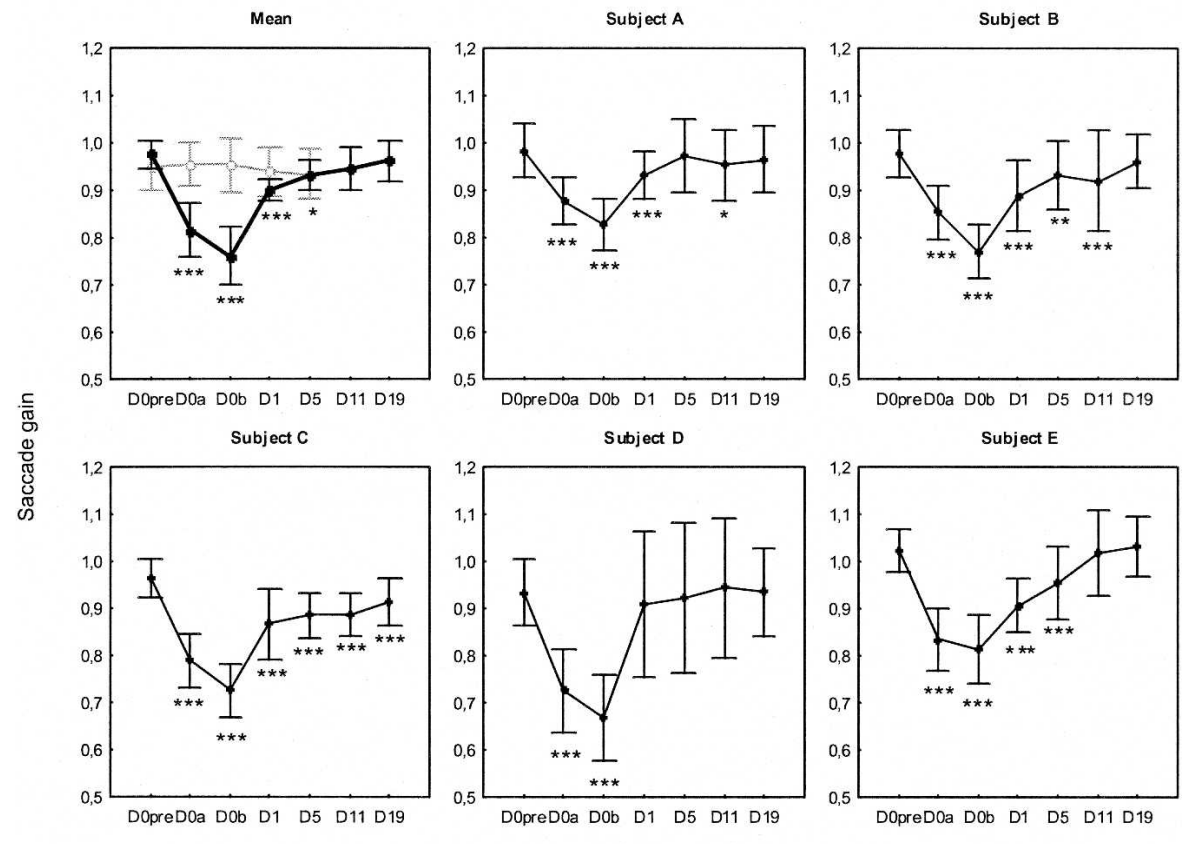

Test sessions

Figure 2. (Top left panel) Mean saccade gain plotted as a function of the test sessions of the main experiment (black line) and of the control experiment (gray line). These test sessions were conducted: (1) on day 0 before (D0pre), during (i.e., after nearly half of the trials; DOa), and immediately after completion of the adaptation (or pseudo-adaptation) session (D0b); (2) on days 1 (D1), 5 (D5); and (3), in the main experiment only, on days 11 (D11) and 19 (D19). The other panels show individual plots of all subjects (A through E) in the main experiment. Error bars, SD. Statistically significant differences of post-test saccade gain with respect to D0pre are indicated by ${ }^{*} P<0.05,{ }^{* *} P<0.01$, and 


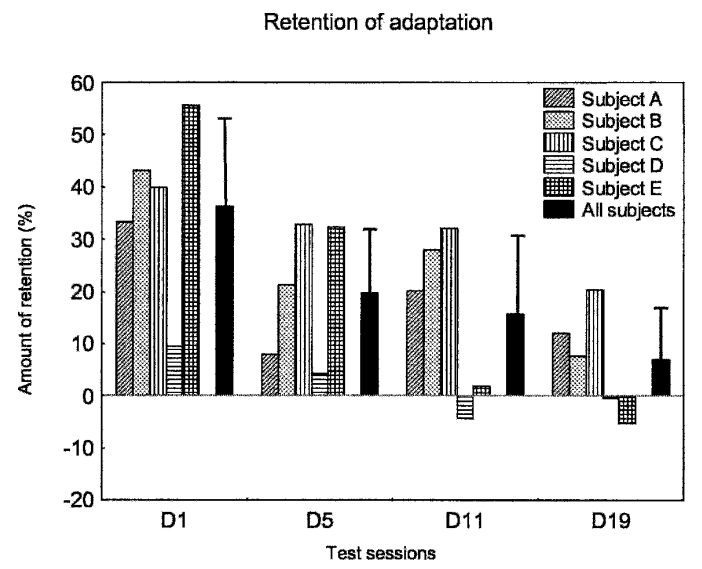

Figure 3. Percentage of retention of adaptation in the main experiment as a function of the test sessions, for each subject $(n=5)$ and for the subjects average (black bars). Error bars, SD.

with a gain change of $-15.6 \% \pm 5 \%$ for the $10^{\circ}$ saccades and of $-17.5 \% \pm 5.1 \%$ for the $20^{\circ}$ saccades. Thus, as depicted in Figure $4 \mathrm{~A}$, the statistically nonsignificant advantage of the $10^{\circ}$ saccades gain relative to the $20^{\circ}$ saccades gain observed at D0pre was maintained throughout, and the time course of adaptationrelated gain change was nearly identical for the two saccade amplitudes. In addition, the amount of retention of adaptation was similar for the two sizes of saccades for all sessions (Fig. 4B). In conclusion, the amounts of adaptation obtained at D0 and retained over the testing period were similar for saccades of $10^{\circ}$ and $20^{\circ}$.

\section{Control experiment}

The control experiment produced results that clearly differed from those of the main experiment. Figure 1B illustrates the gain of all individual primary saccades performed by subject B during the control experiment (day 0). Contrary to the adaptation condition, subject $\mathrm{B}$ did not show in the pseudo-adaptation condition any change in gain over trials or between pre-test and posttest. Note that the same pattern was observed for the four other control subjects (data not shown). As in the main experiment, we performed an ANOVA in each subject who was tested in the control experiment to compare the saccade gain between the different test sessions. Subjects $\mathrm{G}$ and $\mathrm{H}$ did not exhibit any significant main effect $(P>0.6)$ contrary to subjects $\mathrm{B}, \mathrm{C}$, and $\mathrm{F}$ $(P<0.05)$. In these three subjects, a posthoc analysis was performed and revealed a decrease of the gain at D1 and D5 for subject $\mathrm{B}(P<0.001)$, a decrease of the gain at D5 for subject $\mathrm{C}(P<0.05)$, and an increase of the gain at D5 for subject F $(P<0.01)$. But importantly, none of the subjects presented a reduction of the gain at DOa and DOb relative to the pretest. The averaged data across the five subjects are depicted in Figure 2 ("mean", gray trace). The one-way repeated-measures ANOVA comparing the different sessions did not reveal any significant main effect on saccade gain $\left(F_{(4,16)}=0.915 ; P=0.479\right)$, indicating that the saccade gain was equivalent between the different test sessions. Thus, the results of the control experiment suggest that the decrease of saccade gain observed in the main experiment was the consequence of the double-step adaptation protocol and not of uncontrolled factors like fatigue.

\section{Effect of saccade direction}

As both rightward and leftward saccades were exposed during our adaptation protocol, we had the opportunity to examine whether this adaptation affected the gain of rightward and leftward saccades to the same extent.

\section{Time course of adaptation}

The upper trace in Figure 5A represents the time course of the mean saccade gain $(n=5)$ as a function of the blocks of trials during the adaptation session, considering rightward and leftward saccades separately. We can see that the gain evolved in a similar way between the two types of saccades during A blocks. However, beginning with the first B block, the gain of leftward saccades became smaller than the gain of rightward ones. This difference was maintained over the subsequent adaptation blocks. A two-way repeated-measures ANOVA with factors "adaptation block" and "direction" revealed a significant effect of the block factor $(P<0.001)$ as already discussed, no effect of the direction factor $(P=0.23)$, but a significant interaction $\left(F_{(9,36)}=4.68 ; P<0.001\right)$. The post-hoc analysis (Fisher LSD tests) indicated that only during the two A blocks did the gain fail to exhibit a significant difference between the two saccade directions $(P>0.11)$. Thus the gain changes of rightward saccades and of leftward saccades were not identical, indicating that the adaptation of leftward saccades was more efficient.

On the contrary, in the control experiment (Fig. 5A, lower trace), no difference between the two directions could be revealed. The two-way repeated-measures ANOVA (adaptation block $\times$ saccade direction) revealed a significant main effect of the block factor $(P<0.001)$, which is related to the significantly smaller mean saccade gain in the two A blocks than in the following blocks (Fisher LSD tests, $P<0.05$ ). However, there was no significant difference between the two directions $(P=0.31)$ or any interaction $\left(F_{(9,36)}=1.22 ; P=0.31\right)$. Thus, contrary to the results of the main experiment, control data indicated that the relationship of saccade gain as a function of the block of trials was identical for the two saccade directions.

\section{Saccade gain in test sessions}

The relationship of the mean saccade gain $(n=5)$ as a function of the test sessions is plotted for the two saccade directions separately in Figure 5B. In the main experiment (upper traces), the

\section{Learning \& Memory \\ www.learnmem.org}

\section{A- Time course of saccade gain}

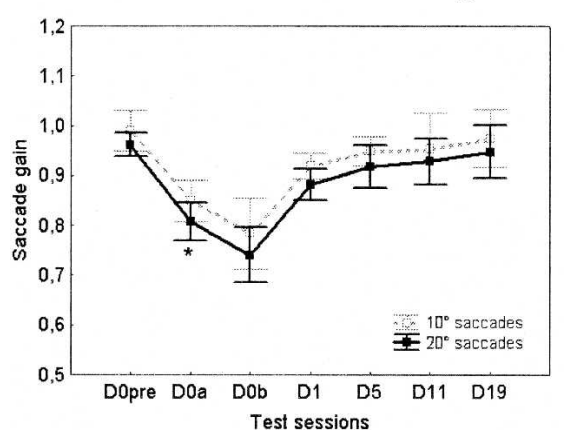

B- Retention of adaptation

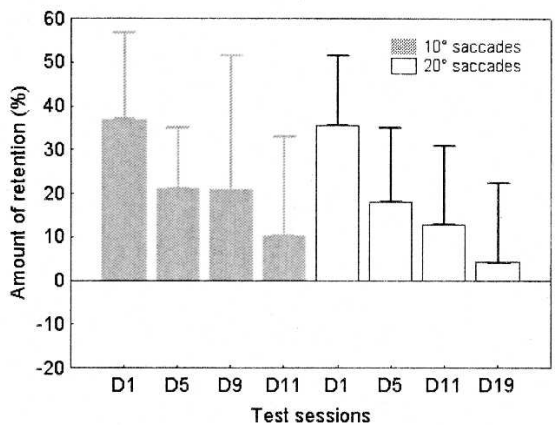

Figure 4. (A) Mean saccade gain plotted separately for the $10^{\circ}$ saccades (dashed gray line) and for the $20^{\circ}$ saccades (black line) as a function of the test sessions of the main experiment (same format as in Fig. 2, "mean"). Error bars, SD. Statistically significant differences of saccade gain between the two types of saccades are shown by $* P<0.05$. (B) Mean percentage of retention of adaptation in the main experiment ( $n=5$ subjects) plotted separately for the $10^{\circ}$ (gray bars) and $20^{\circ}$ (white bars) saccades as a function of the test sessions. Error bars, SD. 
A- Time course of saccade gain

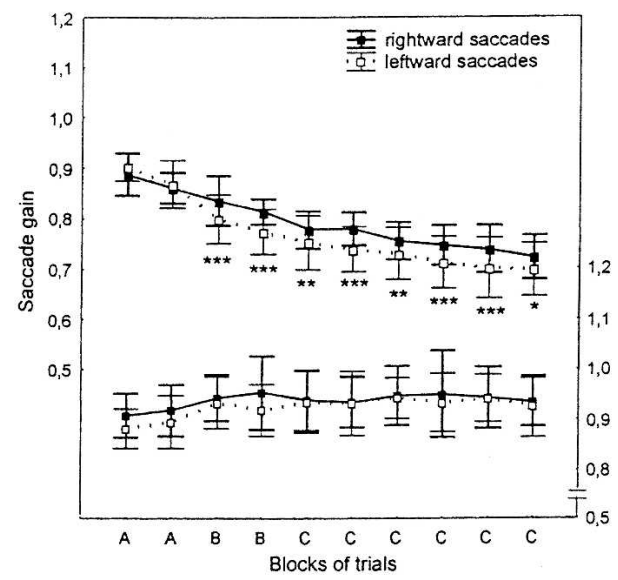

C- Asymmetry of gain (Main)

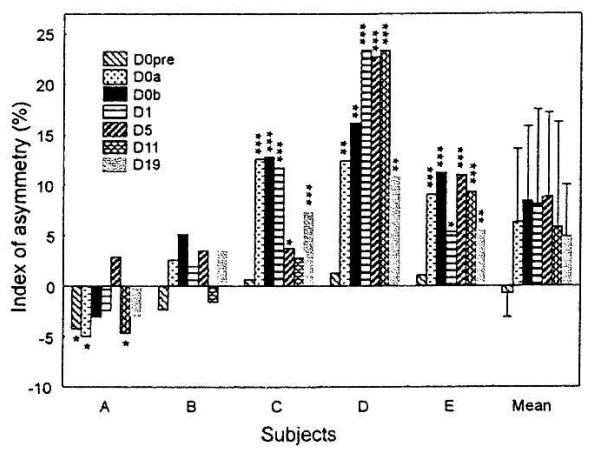

B- Time course of saccade gain

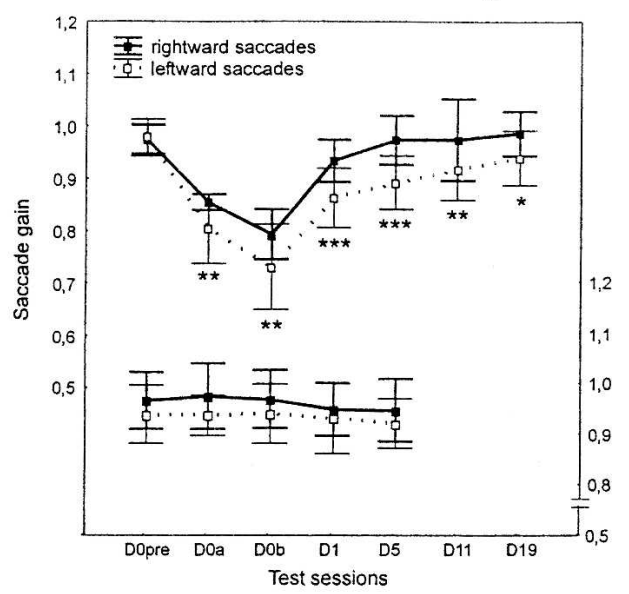

D- Asymmetry of gain (Control)

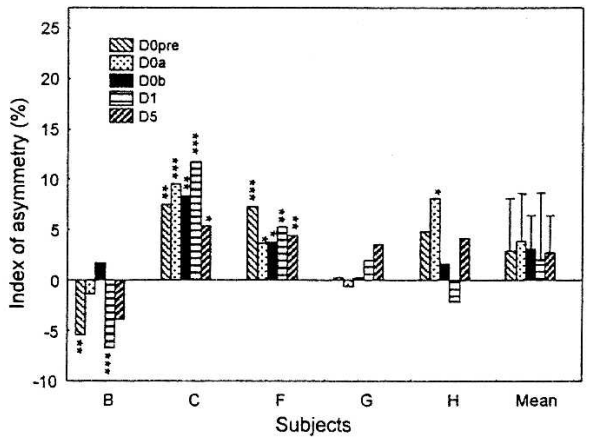

Figure 5. Effect of saccade direction. (A) The gain of rightward (solid line) and of leftward (dotted line) saccades is plotted as a function of the blocks of adaptation trials in the main experiment (upper trace and left $y$-axis) or of pseudo-adaptation trials in the control experiment (lower trace and right $y$-axis). (B) Mean saccade gain plotted separately for the rightward (solid trace) and leftward (dotted trace) direction as a function of the test sessions of the main experiment (upper trace and left $y$-axis; same format as in Figs. 2, 4A), and of the control experiment (lower trace and right $y$-axis). Error bars, SD. (C, D) The IA (\%) of gain according to the saccade direction is plotted as a function of the test sessions for each subject $(n=5)$ and for the subjects' average, in the main experiment $(C)$ and in the control experiment $(D)$. Error bars, SD. In each panel, the asterisks indicate statistically significant differences of saccade gain between the two directions $\left({ }^{*} P<0.05\right.$, ${ }^{* *} P<0.01$, and $\left.{ }^{* * *} P<0.001\right)$.

baseline gain (D0pre) was similar for rightward and leftward saccades. But starting from the first post-test (D0a), the gain of leftward saccades became smaller than that of rightward saccades, and this difference was preserved over days. A two-way repeatedmeasures ANOVA (type of test session $\times$ saccade direction) revealed a significant effect of the test session factor $(P<0.001)$ as already described before, but not of the "saccade direction" factor $(P=0.13)$. However, there was a significant interaction between the two factors $\left(F_{(6,24)}=2.65 ; P<0.05\right)$. Post-hoc Fisher LSD tests indicated that the gain was equivalent between the two directions only in the pre-test $(P=0.70)$, and a statistically significant difference between directions was observed in all post-tests $(P<0.05)$. Furthermore, the leftward saccade gain was still significantly smaller at D19 than the pre-test value $(P<0.05)$, whereas the rightward saccade gain returned to a normal value as early as D5 $(P=0.98)$. Thus, these averaged results suggest that the adaptation effect on leftward saccades was stronger and more enduring. To see if this right-left asymmetry was found in all subjects or only in some of them, we computed an index of asymmetry (IA) as follows: \{[rightward gain - leftward gain]/ $[0.5$ (rightward gain + leftward gain) $]\} \times 100$. A positive IA indicated a higher gain for the rightward direction than for the leftward one, whereas a negative value indicated a superiority of the leftward saccade gain. Figure 5C depicts, for the main experi- ment, the IAs as a function of the test sessions for each subject and for the average across the five subjects. In each subject, the saccade gain was submitted to an ANOVA with test session and saccade direction as factors. The five subjects showed a significant effect of the test session factor $(P<0.001)$ as already described above, and of the saccade direction factor $(P<0.01$; except subject $\mathrm{B}, P=0.08$ ). Subjects $\mathrm{C}, \mathrm{D}$, and $\mathrm{E}$ showed a significant interaction between the two factors $(P<0.05)$ contrary to subjects A and B $(P>0.17)$. Post-hoc Fisher LSD tests (subjects $\mathrm{C}$, $\mathrm{D}, \mathrm{E}$ ) or contrast analyses (subjects A and B) were then performed. The results are illustrated by the asterisks in Figure 5C. Except subjects A and B, the subjects exhibited a significantly higher gain for the rightward saccades than for the leftward saccades as early as the D0a post-test: This right superiority was not present in the D0pre session, was preserved over days and tended to decrease at D19. Thus, these results suggest that, for three subjects out of five, the effect of the double-step target protocol was larger on leftward saccades, and this difference remained over successive days.

Concerning now the control experiment, the difference of gain between the two saccade directions was much less pronounced and remained nearly constant across the successive test sessions. As shown in Figure 5B (lower traces), the gain averaged over the five subjects was slightly lower for leftward saccades 
than for rightward saccades, particularly at DOa and DOb. However, a two-way repeated-measures ANOVA (type of test session $\times$ saccade direction) did not reveal any significant effect of the test session $(P=0.43)$ and of the saccade direction $(P=0.20)$ factors or any significant interaction $\left(F_{(4,16)}=0.32\right.$; $P=0.86)$. ANOVAs comparing the test sessions and the saccade directions were also performed in each subject. As already mentioned above, subjects $\mathrm{G}$ and $\mathrm{H}$ did not exhibit a significant effect of the test session factor $(P>0.62)$ contrary to the other three subjects $(P<0.05)$. Moreover, only subject $\mathrm{G}$ did not exhibit a significant effect of the saccade direction factor $(P=0.258)$. But importantly, none of the subjects showed any significant interaction $(P>0.23)$ except subject B $(P<0.05)$. The post-hoc Fisher LSD tests performed in subject $B$ or the contrast analyses performed in the other four subjects indicated a superiority of the rightward saccade gain only in subjects $\mathrm{C}$ and $\mathrm{F}$ (see stars on the IAs plotted for individual subjects in Fig. 5D). Note however that this superiority of the rightward direction was explained by the asymmetry of gain already present in pre-test. In conclusion, since the between-session changes in the directional asymmetry were absent in the control experiment and followed in the main experiment a time course similar to the overall adaptationrelated gain changes, we can propose that the gain asymmetry between rightward and leftward saccades was inherent to the adaptation phenomenon itself.

\section{Effect of the double-step target paradigm on saccade dynamics and latency}

\section{Changes in duration, peak velocity, and latency}

We investigated whether the large saccade gain modifications induced by the adaptation protocol at D0 were accompanied by any change in saccade duration, saccade peak velocity, or both parameters. Figure 6 illustrates the duration (panel A) and peak velocity (panel B) of saccades recorded on day 0 before and after the adaptation session (black trace). This figure shows that saccade duration decreased after half of the adaptation session (D0a) and decreased further after completion of the adaptation session (D0b). A similar reduction was observed for the saccade peak velocity. A one-way repeated-measures ANOVA applied to duration revealed a significant main effect of the test session factor $\left(F_{(2,8)}=17.4 ; P<0.01\right)$. The post-hoc analysis (Fisher LSD tests) indicated that saccade duration in the pre-test $(57 \pm 5 \mathrm{msec})$ was significantly larger than that at D0a $(53 \pm 4$ msec; $P<0.01)$ and than that at DOb $(51 \pm 3 \mathrm{msec}) ; P<0.001)$. In addition, the dif-

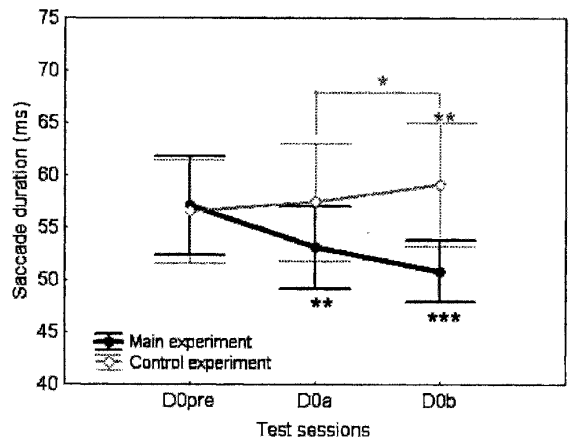

A- Duration
B- Peak velocity

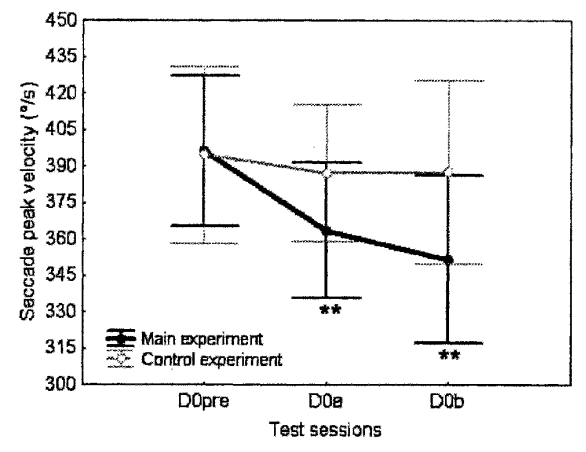

Figure 6. Duration $(A)$ and peak velocity $(B)$ of saccades recorded in the three test sessions on day 0 (D0pre, D0a, D0b) in the main experiment (black trace) and in the control experiment (gray trace). Error bars, SD. Statistically significant differences of saccade parameters between post-test sessions and the D0pre session or between the two post-tests are indicated by ${ }^{*} P<0.05,{ }^{*} P<0.01$, and $* * * P<0.001$. ference of duration between the D0a session and the D0b session failed to reach statistical significance $(P=0.072)$. The same ANOVA applied to the saccade peak velocity showed a significant effect of the test session $\left(F_{(2,8)}=13.6 ; P<0.01\right)$. The post-hoc analysis revealed that peak velocity was significantly larger in pre-test $(396 \pm 32 \mathrm{deg} / \mathrm{sec})$ than in the two post-tests (D0a: $363 \pm 29 \mathrm{deg} / \mathrm{sec}, P<0.01 ;$ D0b: $352 \pm 36 \mathrm{deg} / \mathrm{sec}, P<0.01)$, and again, that saccade peak velocity did not significantly differ between the two post-tests $(P=0.214)$. Latency was also analyzed to determine any change related to the adaptation protocol or to factors such as anticipation or fatigue. The one-way ANOVA showed no effect of test session on latency (mean: $263 \mathrm{msec}$, $\left.F_{(2,8)}=1.15 ; P=0.362\right)$.

In the control experiment, saccade duration and peak velocity were also submitted to a one-way repeated-measures ANOVA with test session as factor (Fig. 6, gray trace). Concerning saccade duration, there was a significant main effect $\left(F_{(2,8)}=7.32\right.$; $P<0.05)$. The post-hoc analysis revealed that the duration at D0a $(57 \pm 6 \mathrm{msec})$ was similar to that at D0pre $(56 \pm 5 \mathrm{msec}$; $P=0.232)$ but that the duration at D0b $(59 \pm 6 \mathrm{msec})$ was significantly longer than that at D0pre $(P<0.01)$. In addition, the duration was larger at D0b than at D0a $(P<0.05)$. Concerning saccade peak velocity, no significant effect was observed (mean: $\left.390^{\circ} / \mathrm{sec} ; F_{(2,8)}=1.18 ; P=0.355\right)$. As in the case of the main experiment, saccade latency was not modified (mean: $237 \mathrm{msec}$; $\left.F_{(2,8)}=0.13 ; P=0.882\right)$. Thus the decrease in duration and peak velocity of saccades observed in the main experiment resulted from the adaptation-related decrease of saccade gain and not from fatigue.

\section{Main sequence relationship}

It is important to determine if these reductions of duration and peak velocity were merely related to the decrease in saccade gain or if they revealed an effect of the adaptation protocol on saccade dynamics. To resolve this question, we analyzed the main sequence relationships. Figure 7 plots the duration versus amplitude relationships for the two subjects who were tested both in the main experiment and in the control experiment (panels $\mathrm{A}$ and B, respectively), for all saccades recorded in the D0pre and DOb sessions. We fitted the relations obtained in the two sessions with a linear function (see Becker 1989). The qualitative analysis of these fits indicates that there was an increase of duration of saccades recorded in post-test with respect to the pre-test data for subject B in both the main and control experiments (top panels). In subject $\mathrm{C}$, however (bottom panels), there was no change in the duration versus amplitude relationship between D0pre and D0b. These two subjects represented the two extreme examples of the spectrum of behavior seen in the eight subjects. We then quantitatively analyzed the relation between saccade duration and amplitude in the whole group of subjects. This relation was first fitted by a linear function in the pre-test session, separately for each subject and for each saccade direction. Then, for each data point collected in the DOb post-test session, we calculated a residual error representing the difference between the actual saccade duration and the duration predicted from the pre-test regression line. Then, the post-test residual errors were averaged separately for each subject and for each saccade direction. The 20 mean residual errors (two groups of five subjects $\times$ two saccade directions) were

\section{Learning \& Memory}

www.learnmem.org 
A- Main experiment

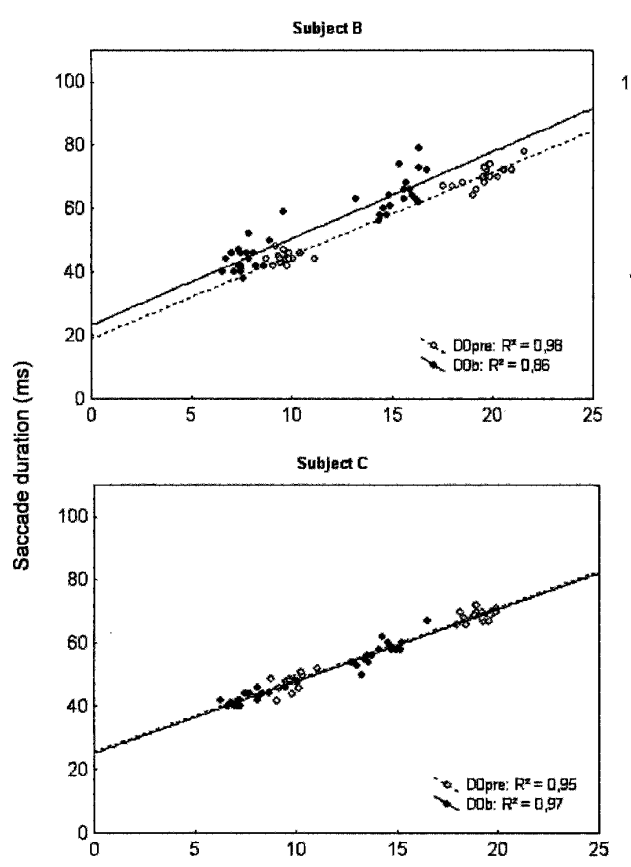

B- Control experiment
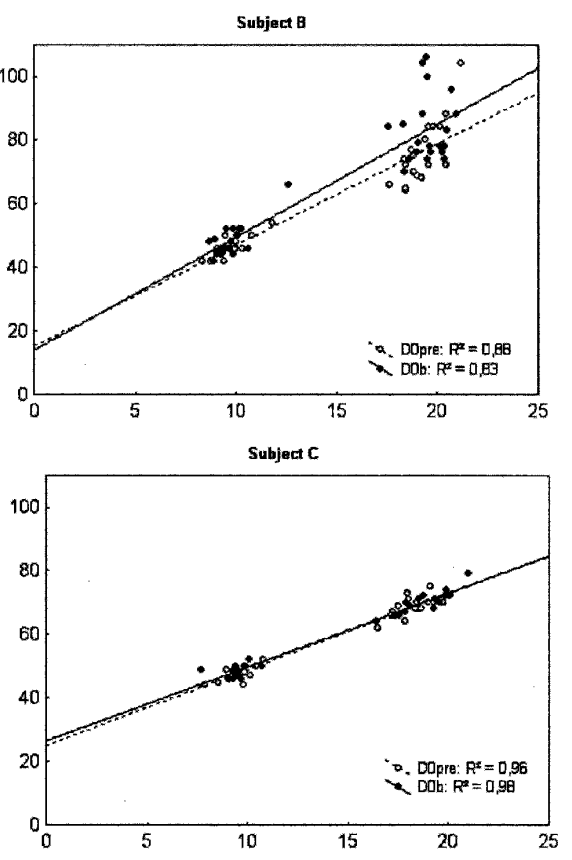

Saccade amplitude (deg)

Figure 7. Main sequence relationships for subjects $B$ and $C$ in the main experiment $(A)$ and in the control experiment $(B)$ at D0. Duration of saccades plotted as a function of their amplitude, in pre-test (open circles) and after the entire adaptation session D0b (filled circles). Each data point represents a saccade, the two directions of saccades being pooled together. Linear relationships represent best-fits through the pre-test (dotted line) and post-test (solid line) data.

submitted to a repeated-measured ANOVA with saccade direction as within-subjects factor and group (main experiment versus control experiment) as between-subjects factor. This statistical analysis did not demonstrate any main effect of the saccade direction factor $(P=0.43)$ or of the group factor $(P=0.53)$ or any significant interaction $\left(F_{(1,8)}=0.26 ; P=0.62\right)$. The data were thus pooled together across the two groups of subjects and the two saccade directions. The overall mean residual error was significantly different from zero $(1.6 \pm 2.4 \mathrm{~ms}$, $n=20$; $t$-test, $P<0.01)$, corresponding to a very small $(2.8 \%)$ increase of the mean saccade duration in the D0b post-test relative to the pre-test. Overall, these analyses indicated that the very slight change in main sequence observed in the post-test relative to the pre-test could not be related to the adaptation phenomenon itself.

The same analyses were performed for the peak velocity versus amplitude relationships, using this time an exponential fit of the pre-test data (see Becker 1989) to compute the post-test residual errors (data not shown). The ANOVA applied to the peak velocity residual error revealed no significant effect of the saccade direction or of the group factors $(P=0.38$ and $P=0.48$, respectively), or any significant interaction $\left(F_{(1,8)}=1.83 ; P=0.21\right)$. The overall mean residual error was negligible $\left(-0.044 \pm 0.24^{\circ}\right)$ sec, corresponding to a $0.011 \%$ decrease of the mean saccade peak velocity in the DOb post-test relative to the pre-test) and did not differ from zero $(n=20 ; t$-test, $P=0.43)$. In conclusion, these peak velocity analyses reinforced the main conclusion of the duration analyses above: The slight increase of saccade duration possibly reflected a moderate development of fatigue, but no change in the saccade dynamic properties could be specifically related to adaptation.

\section{Discussion}

The main objective of our work was to examine if adaptive changes of reactive saccades directed to visual targets persist for some days following the adaptive learning session. Our results showed effectively that adaptation of reactive saccades was partially retained for $5 \mathrm{~d}$. Moreover, both the development and the retention of adaptation were unexpectedly stronger for leftward saccades than for rightward saccades. Our study also showed that the adaptation did not specifically induce any change in the main sequence relationships. We discuss below these different findings.

\section{Retention of saccade gain changes}

Our results showed that on the following day and even up to $5 \mathrm{~d}$ (D5) after adaptation to a backward target step protocol, despite some recovery, the saccade gain remained significantly reduced on average relative to the pre-adaptation level. Thereafter across subsequent tests (D11 and D19), some further-although not statistically significant-recovery has brought the saccade gain back to a pre-adaptation value. However, fatigue or other uncontrolled factors could in principle contribute to day-to-day variations of the gain of saccades, and fatigue may have been especially potent on day 0 after completion of several hundreds of adaptation trials. In fact, as detailed in the Results section, the control experiment allowed us to specifically relate the observed gain changes to the adaptation phenomenon. Thus our study clearly demonstrates that following the double-step protocol, the new acquired saccadic behavior was stored in memory and was partially maintained for days. Maintenance of saccadic adaptation was also reported in monkeys after a 20 -h period in the dark (Straube et al. 1997) or after a 24-h period under normal illumination conditions (Noto et al. 1999). But to our knowledge, our study is the first one to show in humans a retention of saccade adaptation after a recovery period of several days under permanent visual feedback conditions.

It may appear surprising that the adaptive gain changes can persist for such a long time period. Recall that during this delay period, subjects were free to perform their daily activities and were submitted to visual feedback arising from the several thousands of saccades they performed each day. Deubel et al. (1986) observed that when human subjects performed saccades to single-step targets following completion of a double-step target adaptation protocol, de-adaptation (i.e. recovery from adaptation) proceeded with a time course similar to that of the acquisition of adaptation. It can thus be asked why visual feedback failed to completely de-adapt our subjects and to normalize their saccade gain. A likely explanation is that the saccades performed by our subjects everyday differed from those recorded during the test sessions by several aspects. One possibly predominant aspect is the amplitude of the two types of saccades. Indeed, Bahill et al. (1975) have indicated that the amplitude of the majority of saccades generated spontaneously falls well below $15^{\circ}$. Since saccadic adaptation is amplitude specific (Semmlow et al. 1989; Frens and Van Opstal 1994), saccades generated by our subjects 
in between the recording sessions may have been too small to allow the $10^{\circ}$ and $20^{\circ}$ saccades to fully recover from adaptation, especially for the $20^{\circ}$ saccades. In fact, this assumption can be rejected since we found no difference of retention between these saccades and $10^{\circ}$ saccades, suggesting that the adaptation of larger saccades was not better retained. Another factor that may act as a context-cue for saccadic adaptation and that could more likely explain the maintenance of adaptation is the type of saccade initiation: Saccades performed in everyday life are mainly voluntary saccades directed to pre-determined targets, whereas those studied in our protocol are reactive saccades to suddenly presented targets. Indeed, the partial retention of adaptation of reactive saccades found in our study is consistent with the known specificity of adaptation with respect to the type of saccade initiation in humans (Erkelens and Hulleman 1993; Deubel 1995a,b; Fujita et al. 2002; N. Alahyane and D. Pélisson, unpubl.). A context-specificity of adaptation has been further demonstrated recently by Gaveau et al. (2005). These investigators showed that the adaptation of reactive saccades vanished over a 15-min period of presentation of single target steps when subjects performed saccades of the same type (i.e., reactive) but not when they performed voluntary saccades. Finally, several other factors may act as context-cues for saccadic adaptation and may have thus contributed to the long-term persistence of memory for the reactive saccades in our study, including eye position (Alahyane and Pélisson 2004), head position (Shelhamer and Clendaniel 2002), or target distance (Chaturvedi and Van Gisbergen 1997). Which of these factors (including the type of saccade initiation), or combination thereof, contributed most to the observed slowness of de-adaptation cannot be answered from our study. In any case, our study demonstrates in human subjects that when the conditions for de-adaptation are not optimal, adaptive changes of reactive saccades can be maintained in longterm memory.

An unexpected result was that adaptation and its memory were stronger for leftward saccades than for rightward saccades, especially for three out of five subjects. Note that no such difference of amplitude between the two directions was observed in the pre-test session at the start of the experiment, ruling out any baseline difference of gain unrelated to the adaptive process itself. Moreover, the appearance of this difference during the third block of adaptation trials (B block) cannot be easily related to the design of our protocol because (1) the two saccade directions were equally represented in each block, including B blocks; (2) the same number of leftward and rightward trials was performed in each hemifield for all blocks; and (3) in the control experiment, three subjects did not show any difference in gain between leftward and rightward saccades, and the rightward asymmetry found in the remaining two subjects corresponded to a gain difference as early as the pre-test session. In fact, we can only speculate that a minimum number of saccades (>80) and/or a minimum amplitude of error signal (target step- $2=33 \%$ relative to step-1 in B blocks) is required to allow the adaptation process to fully develop and to reveal differences between saccade directions. This difference of robustness of adaptation as a function of the saccade direction was maintained over the long term: Whereas rightward saccades were fully de-adapted as early as D5, leftward saccades remained hypometric until the end of the test period, i.e., after 19 days. In fact, the much longer retention of adaptation of leftward saccades resulted, on the one hand, from the larger gain change observed during the adaptation session and, on the other hand, from the weaker de-adaptation during the delay period. Such asymmetry of gain changes is a much unexpected finding, which to our knowledge has never been reported before. It is well known that an adaptation of a single saccade direction does not affect saccades of the opposite direc- tion in human (Miller et al. 1981; Deubel et al. 1986). This suggests that the adaptation of leftward and rightward saccades relies on separate, and most likely lateralized, neural circuits. Our study further suggests that the neural control of adaptation of leftward saccades is more efficient than that involved in adaptation of rightward saccades. As our trained subjects were all righthanded, it is tempting to propose that the adaptive control of saccadic eye movements may show a stronger contribution of the nondominant hemisphere. This superiority of the right hemisphere has also been invoked, e.g., for the control of attention (Heilman et al. 1985; Coull and Nobre 1998) and of vestibular function (Dieterich et al. 2003). The asymmetry of recovery to a normal saccade gain (i.e., de-adaptation) over the long term may also be explained by a larger efficiency of the nondominant hemisphere in the maintenance of the saccadic motor memory. Another possible reason may be that subjects performed fewer leftward compared with rightward saccades between the recording sessions during tasks such as reading, thus slowing down de-adaptation of saccades in the leftward direction.

\section{Effects of adaptation on saccade dynamics}

Stepping the target backward during a saccade not only produced a decrease in the gain of the saccades but also significantly decreased both their peak velocity and duration. These modifications were, however, not observed in the control experiment, suggesting that they can be specifically related to the adaptation protocol in the main experiment. Another interesting finding was that there was no modification in the main sequence relationships due exclusively to adaptation, suggesting that the decreases of peak velocity and duration of saccades after adaptation could be accounted for merely by the decrease of saccade gain. In other words, our study indicates that adaptation mechanisms primarily acted on the gain and not on the dynamics of saccades. Note that the very weak increase of the saccade duration (main sequence) observed both in the main and in the control experiments, therefore unrelated to adaptation, could have possibly resulted from fatigue, a likely explanation if one considers the large number of trials. There is some controversy in the literature concerning the effect of adaptation on saccade dynamics. Indeed, as in our study, Frens and Van Opstal (1994) found a decrease of both peak velocity and duration without change in the main sequence. In contrast, Abrams et al. (1992) found a change in the main sequence associated with a selective decrease of peak velocity in a backward target step paradigm (their first experiment). One possible explanation of the absence of a decrease of duration in this latter study may be that the adaptation session contained many fewer trials than in our protocol. Straube and Deubel (1995) demonstrated that the forward target step paradigm led to a modification of the dynamics of a "standard $12^{\circ}$ saccade" (decrease in peak velocity and increase in duration) during the adaptation session, whereas the gain decrease paradigm led to a decrease of the peak acceleration/peak deceleration ratio, by comparison with a control condition without intrasaccadic target step. Concerning now adaptation in the monkey, Straube et al. (1997) revealed no average change in saccade dynamics after adaptation but observed a large variability between animals, with the more adapted one exhibiting a slowing of saccade dynamics with an increase of duration and a decrease of peak velocity. Note that cautions have to be taken in the interpretation of the data from the literature since the experiments differ in terms of the number of saccades during the adaptation session, of the amplitude of target steps, or in the choice of the sessions used to compare the saccade dynamical parameters (pre-test, post-test, adaptation, or control session without intrasaccadic step). In our study, the number of saccades and the size of intrasaccadic target step were sufficient to elicit a fairly high level of adaptation, and

\section{Learning \& Memory}


the post- versus pre-test comparison allowed us to minimize effects that were not specifically related to sensori-motor adaptation. The lack of change of saccade dynamical properties observed under these conditions suggests that the plastic neuronal modifications underlying the backward adaptation of reactive saccades do not include the brainstem saccadic pulse generator. Combined with our previous proposal of a collicular or subcollicular locus for saccade adaptation (see discussion in Alahyane et al. 2004), the present work leads us to tentatively suggest that the backward adaptive mechanisms modify a desired eye displacement signal at the collicular level or at the level of reticular formation neurons that transmit collicular signal to the pulse generator.

In conclusion, similar to numerous sensori-motor systems such as visuo-manual tracking (Shadmehr and Holcomb 1997), eyelid conditioning (Medina et al. 2001), or VOR (see for review Boyden et al. 2004), we found, for the saccadic system, that changes of saccade amplitude that quickly develop during a double-step target adaptation protocol can remain in memory for a much longer period of time. Studies of the learning of the former sensori-motor responses showed that short-term changes in motor behavior and their long-term storage may rely on different neural circuits within or outside the cerebellum. By demonstrating the existence of long-term changes of saccadic performance following adaptation, our study raises the possibility to test in the saccadic system such dissociations between neural substrates of the short-term development and of the long-term retention of sensori-motor adaptation.

\section{Materials and Methods}

\section{Subjects}

This study was conducted in eight voluntary subjects (age range: 20-45 yr) after getting their informed consent. All subjects had a normal or a corrected-to-normal vision. Five subjects (A, B, C, D, E) participated in the main experiment. Subjects B and $\mathrm{C}$ also performed the control experiment, together with three other subjects ( $F, G, H)$. Only subjects $B$ and $C$ were not naive about the purpose of this study. The study complied with the declaration of Helsinki.

\section{Apparatus}

Subjects were seated, in a dimly lighted room, in front of a concave spherical board containing red light-emitting diodes (LEDs; diameter, $3 \mathrm{~mm}$ ) used as visual targets. The center of the board was aligned with the subject's naso-occipital axis at $1.10 \mathrm{~m}$ (sphere radius) from the cyclopean eye. The head was stabilized by means of a chin rest. All targets were located along the azimuth at eye level $\left(0^{\circ}\right.$ axis), and their position ranged from $-27.5^{\circ}$ eccentricity (to the left of the center of the board) to $27.5^{\circ}$ (to the right), separated every $2.5^{\circ}$. Two other LEDs used for calibration were situated at $\pm 12.5^{\circ}$ on the vertical axis. Subjects were required to carefully follow the targets with their eyes.

\section{Protocol in main experiment}

\section{Adaptation session}

Saccadic adaptation was induced by the double-step target protocol. At the beginning of a trial, a fixation point was illuminated at a random position on the $0^{\circ}$ axis for a randomly alternating period (1600 msec or $2000 \mathrm{msec}$ ). Then, the fixation point was turned off while a peripheral target (target-1) appeared randomly to the right or to the left (step-1). As soon as the eye velocity exceeded a $50 \%$ sec threshold during the saccadic response toward target-1, the target was displaced again (step-2) to a backward position (target-2). Step-2 was backward relative to step-1 in order to induce a decrease of the saccade amplitude. Each final position of the target in a double-step trial (target-2) became the starting point (fixation point) of the following trial. The adaptation session was composed of three different types of blocks (A, B, and $\mathrm{C}$ ). The A block contained 40 trials, with a target step- 1 amplitude of $10^{\circ}$ or $20^{\circ}$ and a step- 2 amplitude representing $25 \%$ of step-1. In the B block (60 trials), three amplitudes of target displacements were used $\left(7.5^{\circ}, 15^{\circ}\right.$, and $\left.22.5^{\circ}\right)$, and step- 2 amplitude was $33 \%$ of step- 1 . In the C block (40 trials), the amplitude of step- 1 was either $12.5^{\circ}$ or $25^{\circ}$, and the amplitude of the backward jump was $40 \%$ of step- 1 . Note that the three step- 2 amplitudes led us to choose specific step- 1 amplitudes in accordance with the $2.5^{\circ}$ spacing of LEDs along the horizontal axis of our target board. Moreover, we progressively increased step- 2 amplitude in order to minimize the conscious perception of step- 2 by the subjects and to induce a larger adaptation. Indeed, an initial $40 \%$ step- 2 would be consciously detected by subjects who may thus voluntarily compensate for it. Note further that subjects, when asked at the end of experiments, never reported an intrasaccadic step except the two non-naive subjects B and C, who sensed the intrasaccadic step for the $40 \%$ amplitude. In each of the three blocks, the amplitude and direction of step- 1 occurred randomly and were equally represented (10 repetitions). In addition, single-step target trials were inserted within each block (12 trials in A and C blocks; four in the B block) to increase the unpredictability of the whole sequence of target presentation. For these trials, target- 1 was not displaced but was instead turned off during the saccade, and $1800 \mathrm{msec}$ following its presentation, it reappeared at the same position and became the starting point of the following double-step trial. The entire adaptation session consisted of two repetitions of the A block, two repetitions of the $\mathrm{B}$ block, and six repetitions of the C block.

\section{Test sessions}

A test session was performed before the adaptation session (pretest), to measure the initial saccade performances of the subjects, and at different times after the adaptation session (post-tests), to measure the after-effects of saccade adaptation and persistence of memory. In all test sessions, the fixation point was always at the center of the target board. After a random delay (1200 msec, 1800 $\mathrm{msec}$, or $2200 \mathrm{msec}$ ), the fixation point was extinguished and simultaneously a peripheral target (target-1) was illuminated. The peripheral target position varied randomly between trials, either to the right or to the left, at $\pm 10^{\circ}$ or $\pm 20^{\circ}$ eccentricity. In this condition, target- 1 did not step during the saccadic response but instead was turned off to prevent the occurrence of any deadaptation (i.e., recovery from adaptation) during the post-tests. Then, 1800 msec after target- 1 presentation (i.e., 1-1.5 sec after its offset), the next trial was initiated by the presentation of the fixation point at the center of the board. Thus a test session was composed of four types of trials repeated 10 times each.

\section{Experimental schedule}

The adaptation session was performed on day 0 (D0). On day 0 , each subject also performed a pre-test (D0pre) and two post-tests. The first post-test (DOa) was performed after about half of the adaptation session (240 double-step trials: two A blocks, followed by two B blocks, followed by one C block). The second post-test (D0b) was recorded after the remaining 200 adaptation trials were performed (five C blocks). To avoid fatigue in subjects, breaks of 1-2 min, with eyes closed, were inserted between blocks in the adaptation session and between the test and adaptation sessions. These measures on day 0 allowed us to test the efficacy and the immediate after-effects of the adaptation induced by the double-step target protocol.

To test the retention of adaptation, a post-test was run at different times after D0: on days 1 (D1), 5 (D5), 11 (D11), and 19 (D19). Between these different post-tests, the subjects were free to perform their usual daily activities inside and outside the laboratory. Two subjects (subjects $\mathrm{C}$ and $\mathrm{E}$ ) were again tested on day 90 and on day 105, respectively. Indeed, after completion of the data analysis, we noticed that these two subjects exhibited a saccade gain that was still significantly reduced at D19 with respect to D0pre. For subject E, saccade gain was even smaller at D19 
than at D11. The delayed post-tests in these two subjects revealed for subject $\mathrm{E}$ a gain increase at D105, close to the value at D11, suggesting that some uncontrolled factor could be responsible for the gain drop observed at D19 in this subject. For subject C, the gain also increased at D90, even if it remained significantly smaller than the pre-test gain. Thus, for these two subjects, the gain measured at D90 or D105 was substituted for the gain at D19.

\section{Protocol in control experiment}

Subjects B and C performed, respectively, 21 and 32 wk after D0, a control experiment with three other subjects (F, G, and $H)$. The design of the control experiment and the number of trials were exactly the same as in the main experiment. The only difference is that during the adaptation session of the control experiment, eye velocity was not monitored online and no backward target step was induced during saccades. Instead, this second step from target-1 to target- 2 occurred only 1800 msec after target- 1 onset. Target- 2 position then became the fixation point of the following trial. Since the change from target- 1 to target- 2 occurred long after the saccade toward target- 1 was completed ( $>1 \mathrm{sec}$ ), this control protocol should not elicit any sensori-motor adaptation (Shafer et al. 2000; Fujita et al. 2002; see Introduction) but was adequate for testing fatigue effects since the elicited main and corrective saccades were the same as in the main experiment. This session, called pseudo-adaptation, was composed of the same successive blocks as in the adaptation session, with rest periods between blocks. As in the main experiment, this pseudoadaptation session was preceded by a pre-test and followed by a first post-test after five blocks (A, A, B, B, C) and by a second post-test after the last five $C$ blocks, all performed on day 0 . A post-test was also performed on days 1 and 5 (D1 and D5, respectively).

\section{Eye movement recordings and data acquisition}

Horizontal and vertical eye movements were recorded by videooculography using an EyeLink system (SMI), at a frequency of $250 \mathrm{~Hz}$ with an accuracy of $0.1^{\circ}$. A new calibration was performed on each day immediately before recording sessions. During calibration, subjects were required to sequentially look at five targets located at $0^{\circ},-27.5^{\circ}$, and $27.5^{\circ}$ positions on the horizontal axis and at $\pm 12.5^{\circ}$ along the vertical axis.

Left eye position was processed online by an electronic circuit (low pass filtering, differentiation, and thresholding) to generate a signal when the velocity exceeded a threshold of $\sim 50^{\circ} / \mathrm{sec}$. This signal was used by a Data Wave computer program (Berthoud) to trigger the intrasaccadic target step in the adaptation session or to extinguish the target in the test sessions. In addition to target presentation, this computer program sampled eye position data (sampling frequency $=500 \mathrm{~Hz}$ ), displayed eye movements after each trial, and stored the data on disk for off-line analysis.

\section{Data analysis}

The horizontal and vertical components of the movements of the two eyes were analyzed off-line. After filtering $(70 \mathrm{~Hz}$ cut-off frequency, finite impulse response [FIR] filter, residual noise level $<0.5^{\circ}$ ), the initial and final positions of all saccades were marked on the basis of a velocity threshold of $40^{\circ} / \mathrm{sec}$. These two markings were checked by the experimenter and could be manually changed. Trials with a primary saccade latency $<100 \mathrm{msec}$ or $>1000$ msec or trials with a saccade that was not correctly detected online or that was contaminated with a blink were eliminated. For all subjects, excluded trials represented an amount of $8.3 \% \pm 5.9 \%$. For the data analysis, only the saccade in response to step-1, i.e., the primary saccade, was considered. Moreover, we computed the data of the cyclopean eye as the average of the data of the right eye and of the data of the left eye. This choice was dictated by the fact that there were significant differences in gain between rightward and leftward saccades in the pre-test when each eye was analyzed separately (i.e., a larger gain for rightward versus leftward saccades for the left eye and the opposite gain asymmetry for the right eye). To avoid these baseline, eyedependent, directional asymmetries of gain, which would have interfered with our analysis on the effect of saccade direction in saccade adaptation, we averaged the data of the two eyes.

The parameters described in the following were computed for each primary saccade. Saccade amplitude was computed as the difference between the initial and final positions of the eye, and saccade duration was obtained as the corresponding time difference. Saccade gain was calculated as the ratio between saccade amplitude and target step- 1 amplitude. For each subject, the percentage of gain change related to the adaptation protocol was calculated as follows: [(mean post-test gain (at D0a, D0b, D1, D5, D11, or D19) - mean pre-test gain (D0pre))/mean pre-test gain] * 100. Further, in the main experiment, the amount of retention was computed as follows: [percentage gain change (at D1, D5, D11, or D19)/percentage gain change at D0b] * 100. In a first part of the Results section, data of rightward and leftward saccades were pooled together. In a second part, rightward and leftward saccades were differentiated in order to test whether the adaptation equally affected both directions.

For the main experiment, the duration, peak velocity and latency of saccades recorded at D0 were compared between the pre-test (D0pre) and the two post-tests (D0a, DOb) by a repeatedmeasures ANOVA with the within-subjects test session factor ( $n=5$ subjects). Concerning saccade amplitude, the pre-test (D0pre) gain was compared to the post-test gain recorded on different days (D0a, D0b, D1, D5, D11, D19) by using a repeatedmeasures ANOVA with test session as the within-subjects factor. We then investigated if saccade gain in test sessions could differ according to the amplitude of the target displacement $\left(10^{\circ}\right.$ and $20^{\circ}$ ) by a repeated-measures ANOVA with two factors (test session and target amplitude). Similarly, the difference in saccade gain according to the leftward versus rightward direction of saccades was tested by a repeated-measures ANOVA with two factors (test session and saccade direction). For the control experiment, the same comparisons between the pre-test and the post-tests as those described above were made, and the statistical analyses used the same repeated-measures ANOVAs as in the main experiment ( $n=5$ subjects). Post-hoc comparisons were performed by means of Fisher LSD tests. Statistical significance level was set at $P<0.05$.

\section{Acknowledgments}

We thank all the subjects for their participation in the study. We also thank Christian Urquizar and Marcia Riley for designing the data replay/parameter extraction software. This work was supported by INSERM U534.

\section{References}

Abel, L.A., Schmidt, D., Dell'Osso, L.F., and Daroff, R.B. 1978. Saccadic system plasticity in humans. Ann. Neurol. 4: 313-318.

Abrams, R.A., Dobkin, R.S., and Helfrich, M.K. 1992. Adaptive modification of saccadic eye movements. J. Exp. Psychol. Hum. Percept. Perform. 18: 922-933.

Alahyane, N. and Pélisson, D. 2004. Eye position specificity of saccadic adaptation. IOVS 45: 123-130.

. 2005. Retention of saccadic eye movements. Ann. NY Acad. Sci. 1039: 558-562.

Alahyane, N., Koene, A., and Pélisson, D. 2004. Transfer of adaptation from visually-guided saccades to averaging saccades elicited by double visual targets. Eur. J. Neurosci. 20: 827-836.

Albano, J.E. 1996. Adaptive changes in saccade amplitude: Oculocentric or orbitocentric mapping? Vis. Res. 36: 2087-2098.

Bahcall, D.O. and Kowler, E. 2000. The control of saccadic adaptation: Implications for the scanning of natural visual scenes. Vis. Res. 40: $2779-2796$.

Bahill, A.T., Clark, M.R., and Stark, L. 1975. The main sequence: A tool for studying human eye movements. Math. Biosci. 24: 191-204.

Barash, S., Melikyan, A., Zhang, M., Glickstein, M., and Thier, P. 1999. Saccadic dysmetria and adaptation after lesions of the cerebellar cortex. J. Neurosci. 19: 10931-10939.

Becker, W. 1989. Metrics. In The Neurobiology of saccadic eye movements (eds. R.H. Wurtz and M.E. Goldberg), pp. 13-67. Elsevier, Amsterdam.

Boyden, E.S., Katoh, A., and Raymond, J.L. 2004. Cerebellum-dependent 
learning: The role of multiple plasticity mechanisms. Annu. Rev. Neurosci. 27: 581-609.

Caithness, G., Osu, R., Bays, P., Chase, H., Klassen, J., Kawato, M. Wolpert, D.M., and Flanagan, J.R. 2004. Failure to consolidate the consolidation theory of learning for sensorimotor adaptation tasks. J. Neurosci. 24: 8662-8671.

Chaturvedi, V. and Van Gisbergen, J.A. 1997. Specificity of saccadic adaptation in three-dimensional space. Vis. Res. 37: 1367-1382.

Coull, J.T. and Nobre, A.C. 1998. Where and when to pay attention: The neural systems for directing attention to spatial locations and to time intervals as revealed by both PET and fMRI. J. Neurosci. 18: $7426-7435$.

Desmurget, M., Pélisson, D., Urquizar, C., Prablanc, C., Alexander, G.E., and Grafton, S.T. 1998. Functional anatomy of saccadic adaptation in humans. Nat. Neurosci. 1: 524-528.

Deubel, H. 1987. Adaptivity of gain and direction in oblique saccades. In Eye movements: From physiology to cognition (eds. J.K. O'Regan and A. Levy-Schoen), pp. 181-190. Elsevier, New York.

. 1995a. Separate adaptative mechanisms for the control of reactive and volitional saccadic eye movements. Vis. Res. 35: 35293540 .

- 1995b. Is saccade adaptation context-specific? In Eye movement research: Mechanisms, processes and applications (eds. J.M. Findlay, et al.), pp. 177-187. Elsevier Science, Amsterdam.

Deubel, H., Wolf, W., and Hauske, G. 1986. Adaptive gain control of saccadic eye movements. Hum. Neurobiol. 5: 245-253.

Dieterich, M., Bense, S., Lutz, S., Drzezga, A., Stephan, T., Bartenstein, P., and Brandt, T. 2003. Dominance for vestibular cortical function in the non-dominant hemisphere. Cereb. Cortex 13: 994-1007.

Erkelens, C.J. and Hulleman, J. 1993. Selective adaptation of internally triggered saccades made to visual targets. Exp. Brain Res. 93: 157164.

Fernandez-Ruiz, J. and Diaz, R. 1999. Prism adaptation and aftereffect: Specifying the properties of a procedural memory system. Learn. Mem. 6: $47-53$.

Frens, M.A. and Van Opstal, A.J. 1994. Transfer of short term adaptation in human saccadic eye movements. Exp. Brain Res. 100: 293-306.

Fujita, M., Amagai, A., Minakawa, F., and Aoki, M. 2002. Selective and delay adaptation of human saccades. Cogn. Brain Res. 13: 41-52.

Gaveau, V., Alahyane, N., Salemme, R., and Desmurget, M. 2005. Self-generated saccades do not modify the characteristics of adaptive reactive saccades. Exp. Brain Res. 162: 526-531.

Heilman, K.M., Watson, R.T., and Valenstein, E. 1985. Neglect and related disorders. In Clinical neuropsychology (eds. K.M. Heilman and E. Valenstein), pp. 243-293. Oxford University Press, New York.

Hopp, J.J. and Fuchs, A.F. 2004. The characteristics and neuronal substrate of saccadic eye movements plasticity. Prog. Neurobiol. 72: $27-53$.

Kommerell, G., Olivier, D., and Theopold, H. 1976. Adaptive programming of phasic and tonic components in saccadic eye movements: Investigations of patients with abducens palsy. Invest. Opthalmol. 15: 657-660.
Kuki, Y., Hirata, Y., Blazquez, P.M., Heiney, S.A., and Highstein, S.M. 2004. Memory retention of vestibuloocular reflex motor learning in squirrel monkeys. Neuroreport 15: 1007-1011.

McLaughlin, S.C. 1967. Parametric adjustment in saccadic eye movements. Percept. Psychophys. 2: 359-362.

Medina, J.F., Garcia, K.S., and Mauk, M.D. 2001. A mechanism for savings in the cerebellum. J. Neurosci. 21: 4081-4089.

Miller, J.M., Anstis, T., and Templeton, W.B. 1981. Saccadic plasticity: Parametric adaptive control by retinal feedback. J. Exp. Psychol. 7: 356-366.

Noto, C.T., Watanabe, S., and Fuchs, A.F. 1999. Characteristics of simian adaptation fields produced by behavorial changes in saccade size and direction. J. Neurophysiol. 81: 2798-2813.

Optican, L.M. and Robinson, D.A. 1980. Cerebellar-dependent adaptative control of primate saccadic system. J. Neurophysiol. 44: $1058-1076$.

Scudder, C.A., Batourina, E.Y., and Tunder, G.S. 1998. Comparison of two methods of producing adaptation of saccade size and implications for the site of plasticity. J. Neurophysiol. 79: 704715 .

Semmlow, J.L., Gauthier, G.M., and Vercher, J.L. 1989. Mechanisms of short-term saccadic adaptation. J. Exp. Psychol. Hum. Percept. Perform. 15: $249-258$.

Shadmehr, R. and Brashers-Krug, T. 1997. Functional stages in the formation of human long-term motor memory. J. Neurosci. 17: 409_ 419.

Shadmehr, R. and Holcomb, H.H. 1997. Neural correlates of motor memory consolidation. Science 277: 821-825.

Shafer, J.L., Noto, C.T., and Fuchs, A.F. 2000. Temporal characteristics of error signals driving saccadic gain adaptation in the macaque monkey. J. Neurophysiol. 84: 88-95.

Shelhamer, M. and Clendaniel, R. 2002. Sensory, motor, and combined contexts for context-specific adaptation of saccade gain in humans. Neurosci. Lett. 332: 200-204.

Straube, A. and Deubel, H. 1995. Rapid gain adaptation affects the dynamics of saccadic eye movements in humans. Vis. Res. 23/24: 3451-3458.

Straube, A., Fuchs, A.F., Usher, S., and Robinson, F.R. 1997. Characteristics of saccadic gain adaptation in rhesus macaques. J. Neurophysiol. 77: 874-895.

Straube, A., Deubel, H., Ditterich, J., and Eggert, T. 2001. Cerebellar lesions impair rapid saccade amplitude adaptation. Neurology 57: $2105-2108$

Tong, C. and Flanagan, J.R. 2003. Task-specific internal models for kinematic transformations. J. Neurophysiol. 90: 578-585.

Watanabe, S., Noto, C.T., and Fuchs, A. 2000. Flexibility of saccade adaptation in the monkey: Different gain states for saccades in the same direction. Exp. Brain Res. 130: 169-176.

Received January 4, 2005; accepted in revised form June 14, 2005. 


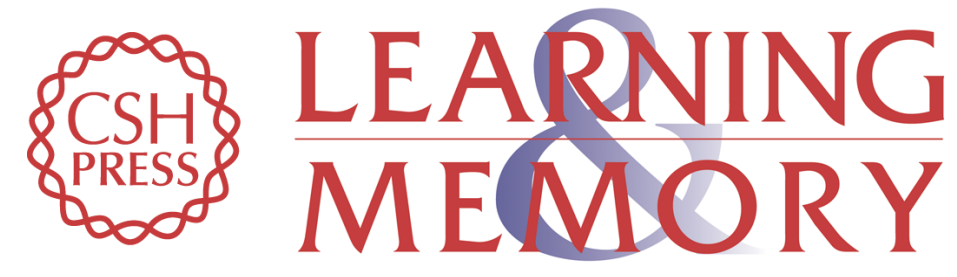

\section{Long-lasting modifications of saccadic eye movements following adaptation induced in the double-step target paradigm}

Nadia Alahyane and Denis Pélisson

Learn. Mem. 2005, 12:

Access the most recent version at doi:10.1101/lm.96405

References This article cites 40 articles, 9 of which can be accessed free at: http://learnmem.cshlp.org/content/12/4/433.full.html\#ref-list-1

License

Email Alerting

Receive free email alerts when new articles cite this article - sign up in the box at the Service top right corner of the article or click here. 